\title{
PERFIL DOS ESTUDOS DE ASSISTÊNCIA DE ENFERMAGEM AO PORTADOR DE TUBERCULOSE NO BRASIL
}

\author{
PROFILE OF NURSING CARE STUDIES FOR TUBERCULOSIS \\ PATIENTS IN BRAZIL
}

\author{
Vitória Keller Gregorio de Araújo ${ }^{1}$ \\ Sandy Yasmine Bezerra e Silva ${ }^{2}$ \\ Vitória Daeny Medeirosdo Nascimento ${ }^{3}$ \\ Érika Simone Galvão Pinto ${ }^{4}$
}

\begin{abstract}
RESUMO: OBJETIVO: Descrever o perfil dos estudos de assistência de enfermagem ao portador detuberculose produzidos no Brasil. MÉTODO: Trata-se de um estudo de revisão de literatura, descritivo e quantitativo. A busca dos estudos foi realizada no mês de dezembro de 2019, através da Biblioteca Digital Brasileira de Teses e Dissertações. RESULTADOs: foram selecionados 11 estudos para integrarem a amostra. Os estudos selecionados foram produzidos entre os anos 2012 e 2019. Em relação ao tipo do estudo, 72,72\% eram dissertações de mestrado. Identificou-se a prevalência de estudos sobre o tratamento e avaliação do controle da TB. CONCLUSÃO: Os estudos sobre assistência de enfermagem ao portador de TB produzidos no Brasil focalizaram aspectos do tratamento e da avaliação em saúde. Esses achadoscorroboram com as principais dificuldades encontradas no cenário brasileiro frente à doença.
\end{abstract}

Palavras chave: Enfermagem. Revisão. Tuberculose.

ABSTRACT: OBJECTIVE: To describe the profile of nursing care studies for tuberculosis patients produced in Brazil. METHOD: This is a descriptive and quantitative literature review study. The study was conducted in December 2019 through the Brazilian Digital Library of Theses and Dissertations. RESULTS: 11

\footnotetext{
${ }^{1}$ Graduanda do curso de Enfermagem da Universidade Federal do Rio Grande do Norte.

2 Mestre em Enfermagem pelo Programa de Pós Graduação em Enfermagem da Universidade Federaldo Rio Grande do Norte.

${ }^{3}$ Graduanda do curso de Enfermagem da Universidade Federal do Rio Grande do Norte.

${ }^{4}$ Doutora em Enfermagem em Saúde Pública. Professora do Departamento de Enfermagem da Universidade Federal do Rio Grande do Norte.
} 
studies were selected to be part of the sample. The selected studies were produced between 2012 and 2019. Regarding the type of study, 72.72\% were master's dissertations. The prevalence of studies on the treatment and evaluation of TB control was identified. CONCLUSION: Studies on nursing care for TB patients produced in Brazil focused on aspects of treatment and health evaluation. These findings corroborate the main difficulties encountered in the Brazilian scenario regarding the disease.

Keywords: Nursing. Review. Tuberculosis. 


\section{INTRODUÇÃO}

A tuberculose (TB) é caracterizada como uma doença infecciosa vinculada, principalmente, aos males que afetam o pulmão, no entanto, pode manifestar-se clinicamente de diversas maneiras e em órgãos variados (KOZAKEVICH, 2015) podendo ser do tipo pulmonar ou extrapulmonar.

Apesar da descoberta dessa doença ter ocorrido há muitos anos, é ainda considerada um problema de saúde pública, tendo em vista o número de casos no mundo. E isso se deve a vários fatores como a pobreza, desigualdade social, inadequação do tratamento aos novos casos, poucas informações sobre a doença e a falta de políticas públicas e gestão efetiva de combate à transmissão da doença (RODRIGUES, 2010).

Em 2017 foi estimado que 10 milhões de pessoas adoeceram por TB e cerca de 1,3 milhões de pessoas no mundo morreram pelo mesmo motivo. No Brasil, em 2018, estima-se que ocorreram 72.788 mil novos casos da doença o que corresponde a uma incidência de aproximadamente 35 casos a cada 100 mil habitantes, sendo os estados do Amazonas e Rio de Janeiro aqueles que apresentaram maior incidência de casos (BRASIL, 2019).

No entanto, apesar dos números alarmantes de incidência da doença os constantes casos de subnotificação mascaram os reais valores e abrem portas para que haja sub- diagnósticos. Em 2017, das 10 milhões de pessoas que adoeceram por tuberculose, apenas 6,3 milhões foram oficialmente registradas no sistema de vigilância, deixando cerca de 3,6 milhões de pessoas não diagnósticas ou detectadas e não registradas.

Nesse contexto, existem obstáculos enfrentados no controle da TB, seja a subnotificação dos casos, que afeta substancialmente a cobertura de diagnósticos e prevenção (OMS, 2018), como também o abandono do tratamento, o qual se estima que haja uma taxa deabandono em cerca de $40 \%$, enquanto o aceitável seria em 
torno de $5 \%$. As consequênciasgeradas incluem aumento do custo do tratamento, da mortalidade e das taxas derecidiva (RODRIGUES, 2010).

Diante disso, é plausível reafirmar a importância da equipe de saúde no controle da doença, e a enfermagem, inserida na equipe, estabelece um papel promissor. Estudos verificaram que os profissionais de enfermagem têm contribuído para o controle da TBexercendo atividades assistenciais, gerenciamento dos casos, atividades relacionadas aos sistemas de informação em saúde e no desenvolvimento de atividades de educação em saúde (BRUNELLO et. al, 2015; RÊGO, et. al, 2015).

Estudos relacionados à assistência de enfermagem ao portador de TB são importantes para fomentar a maneira como o controle da doença está sendo implementada por essacategoria e como a população pode ser beneficiada, além de analisar de que forma o conhecimento cientifico está sendo pautado, uma vez que é importante para a implementação das boas práticas de enfermeiros e demais profissionais da saúde.

Esse estudo tem como objetivo descrever o perfil dos estudos de assistência de enfermagem ao portador de tuberculose produzidos no Brasil.

\section{MÉTODOS}

Trata-se de um estudo de revisão de literatura, descritivo e quantitativo. A busca dos estudos foi realizada no mês de dezembro de 2019, através da Biblioteca Digital Brasileira de Teses e Dissertações. A questão norteadora consistiu em: qual o perfil dos estudos de assistência de enfermagem ao portador de TB produzido no Brasil?

Como estratégias de investigação foram utilizados os Descritores em Ciências da Saúde (DeCS): "Tuberculose" e "Enfermagem". Foram incluídos estudos publicados entre os anos de 2009 e 2019, disponíveis na íntegra para acesso gratuito e que abordassem a temática proposta. Excluíram-se estudos que 
abordassem coinfeccção TB/HIV e estudos que não correspondessem à temática relevante ao objetivo.

A primeira seleção dos estudos se deu pela leitura dos títulos; em seguida, da leitura dos resumos e as informações obtidas foram apresentadas na forma de tabela, com posterior categorização dos dados.

Para análise das informações foi utilizada uma planilha criada no softwareMicrosoft Excel ${ }^{\circledR}$ composta pelos itens: autor, ano de publicação, tipo de abordagem, temática e temáticaabordada.

\section{RESULTADOS}

Foram encontrados 231 estudos. A partir da análise de seus títulos foram selecionados $13 \mathrm{e}$, posterior à leitura de seus resumos, foram selecionados 11 estudos para integrarem a amostra.A descrição dos estudos selecionados é apresentada no Quadro 1. 
Quadro 1 - Descrição dos estudos sobre assistência de enfermagem ao portador de tuberculose produzidos no Brasil segundo autor, ano de publicação, tipo de abordagem,temática e temática abordada, Natal, Brasil, 2019.

\begin{tabular}{|c|c|c|c|c|}
\hline Autor & Ano & Tipo & Abordagem & Temática \\
\hline $\begin{array}{c}\text { Orlandi, Giovanna } \\
\text { Mariah. }\end{array}$ & 2016 & Dissertação & Misto & Tratamento \\
\hline $\begin{array}{l}\text { Vieira, Alexandre } \\
\text { Bernardini. }\end{array}$ & 2016 & Dissertação & Qualitativa & Busca ativa de casos \\
\hline $\begin{array}{c}\text { Pinheiro, Patrícia } \\
\text { Geórgia Oliveira Diniz. }\end{array}$ & 2016 & Tese & Quantitativa & $\begin{array}{c}\text { Dificuldades no } \\
\text { controle da doença }\end{array}$ \\
\hline $\begin{array}{c}\text { Silva, Sandy Yasmine } \\
\text { Bezerra e. }\end{array}$ & 2018 & Dissertação & Quantitativa & $\begin{array}{l}\text { Avaliação docontrole } \\
\text { da Tuberculose }\end{array}$ \\
\hline $\begin{array}{l}\text { Costa, Tatiana Castro } \\
\text { da. }\end{array}$ & 2019 & Dissertação & Quantitativa & $\begin{array}{c}\text { Avaliação docontrole } \\
\text { daTuberculose }\end{array}$ \\
\hline $\begin{array}{c}\text { Figueiredo, Júlia } \\
\text { Borges. }\end{array}$ & 2018 & Dissertação & Qualitativa & Tratamento \\
\hline $\begin{array}{l}\text { Maria Fernanda } \\
\text { Simiele Beck. }\end{array}$ & 2015 & Dissertação & Quantitativa & Tratamento \\
\hline $\begin{array}{c}\text { Souza, Sabrina da } \\
\text { Silvade. }\end{array}$ & 2012 & Tese & Qualitativa & $\begin{array}{c}\text { Apoio ao portadorde } \\
\text { Tuberculose }\end{array}$ \\
\hline $\begin{array}{c}\text { Oliveira, Déborah } \\
\text { Raquel Carvalho de. }\end{array}$ & 2018 & Tese & Qualitativa & Educação permanente \\
\hline $\begin{array}{c}\text { Oliveira, Déborah } \\
\text { Raquel Carvalho de. }\end{array}$ & 2014 & Dissertação & Quantitativa & $\begin{array}{l}\text { Consulta de } \\
\text { enfermagem }\end{array}$ \\
\hline $\begin{array}{c}\text { Andrade, Séfora } \\
\text { LuanaEvangelista de }\end{array}$ & 2014 & Dissertação & Qualitativa & Tratamento \\
\hline
\end{tabular}

Fonte: Elaboração dos autores (2020).

Os estudos selecionados foram produzidos entre os anos 2012 e 2019, com os maiorespercentuais em 2016 e 2018 (ambos com 27,27\%).

Em relação ao tipo do estudo, $72,72 \%$ eram dissertações de mestrado. Quanto à abordagem utilizada, 05 estudos eram quantitativos $(45,45 \%), 05$ qualitativos $(45,45 \%)$ e apenas 01 utilizou a abordagem mista (10\%). O gráfico 1 apresenta o percentual dos estudosem relação à temática. 
Gráfico 01 - Estudossobre assistência de enfermagem ao portador de tuberculose produzidono Brasil segundo a temática.

$$
50,00 \%
$$

$45,00 \%$

$40,00 \%$

$35,00 \%$

$30,00 \%$

$25,00 \%$

$20,00 \%$

$15,00 \%$

$10,00 \%$

$5,00 \%$

$0,00 \%$

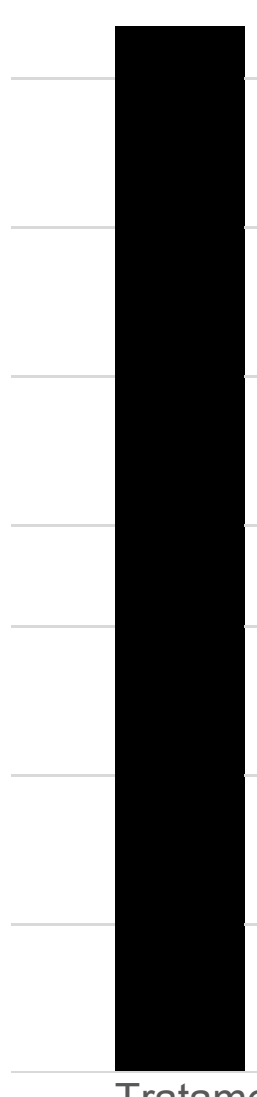

Tratamento

Avaliação de controle daDemais estudos com TB

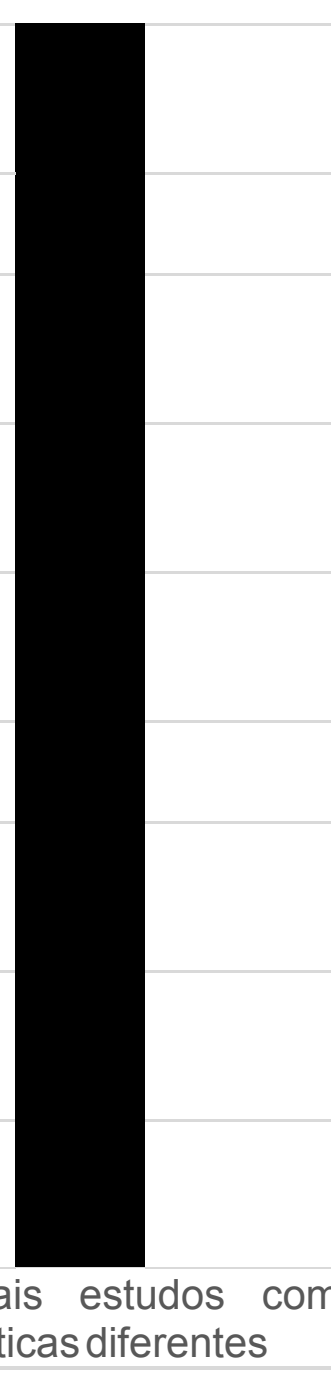

Fonte: Elaboração dos autores, 2020. 


\section{DISCUSSÃO}

A análise dos estudos permitiu verificar que a maioria das pesquisas sobre assistência de enfermagem ao portador de TB produzidos no Brasil se detém ao tratamento da doença.

Foram identificados estudos sobre as dificuldades encontradas tanto pelos profissionais como os usuários na conclusão do tratamento, bem como sobre estratégias para efetivação do Tratamento Diretamente Observado.

A terapêutica utilizada em combate à infecção de tuberculose tem por objetivo a redução da carga bacilar (com foco em evitar transmissão), prevenir contra cepas que são naturalmente resistentes para evitar que haja ineficácia durante o uso dos medicamentos e esterilizar a lesão para evitar que ocorra recidiva. A eficácia desse tratamento está em torno de $95 \%$, no entanto, os obstáculos encontrados abaixam esse quadro para somente $75 \%$ daqueles pacientes que chegam até final do tratamento (RABAHI et. al., 2017).

Silva (2016) relata sobre as condições associadas ao uso dos medicamentos que provocam efeitos colaterais nocivos nos pacientes como um dos fatores que intervém na cura da doença. Devido a este fato, muitos pacientes que já estão debilitados em decorrência da infecção alegam agregar dores epigástricas, náuseas e cefaleia como os sintomas mais frequentes e por isso acabam abandonando o tratamento.

Nesse sentido, os profissionais da área da saúde que lidam diretamente com esses usuários precisam estar atentos, oferecer oportunidades e possibilidades para o fortalecimento do tratamento e a participação da enfermagem, historicamente, trouxe para a categoria um papel fundamental do cuidado além da entrega de medicamentos e realização de exames. É necessário que haja envolvimento com o usuário, orientação e compreensão das dificuldades enfrentadas ao longo de sua vida e que podem influenciar o curso do tratamento (CAVALCANTE et. al., 2016).

Através da análise identificaram-se estudos sobre a avaliação em saúde no contexto da TB. Mesmo em um quantitativo reduzido desses estudos é possível 
estabelecer uma perspectiva de desenvolvimento dessas pesquisas para a busca de informações e consequente tomada de decisão pelos gestores de saúde.

Por isso, o compromisso profissional de controle da tuberculose deve envolver ações necessárias específicas para lidar com o cuidado à pessoa com TB. Esses conhecimentos são adquiridos ao longo da carreira e devem ser fruto de investimento pessoal e profissional que devem ter domínio técnico, que seja respaldado em conhecimentos científicos para fundamentar a prática para que o enfermeiro ou qualquer outro profissional de saúde possa lidar com os problemas que são específicos da patologia (CAVALCANTE et. al. 2016).

Entretanto, os serviços de saúde no Brasil não estão aptos o suficiente para lidar com as demandas que chegam as unidades de saúde e isso acarreta em falha para eliminação da doença já que existem problemas em relação à integração das redes de saúde, baixa resolutividade do diagnóstico e também profissionais não capacitados para assistir ao portadorde TB (ANDRADE et. al., 2017).

O Programa Nacional de Controle da Tuberculose estipula a uniformidade no controle da doença, e visa principalmente aumentar a adesão dos usuários ao tratamento e a cura para reduzir o número de infectados. Essa ação aumentou o acesso dos mais necessitados e estabeleceram avaliações para o controle da TB, além de estruturarem as redes de Atenção Primária à Saúde que oferecem o primeiro contato com o portador (ANDRADE et. al., 2017).

Dessa forma, é essencial que o tratamento e a avaliação do controle da tuberculose sejam interpretados para além dos estigmas que foram gerados ao longo da história. Ou seja, para que haja diminuição da quantidade de infectados deve haver participação coletiva de profissionais que enxerguem a necessidade de se capacitarem e auxiliarem seus pacientes, de usuários que se comprometam em seguir o tratamento conforme recomendado e do poder público que deve oferecer subsídios para as redes de saúde implementarem as ações de controle necessárias. 


\section{CONCLUSÃO}

Os estudos sobre assistência de enfermagem ao portador de TB produzidos no Brasil focalizaram aspectos do tratamento e da avaliação em saúde. Esses achados corroboram com as dificuldades relacionadas ao abandono do tratamento, à implementação dos programas de TB encontradas no cenário brasileiro frente à doença.

Como limitações do estudo há a quantidade reduzida de publicações encontradas, pois pelo tempo delimitado esperava-se que houvesse um número maior de escritos em decorrência da incidência dos casos de tuberculose no Brasil.

\section{REFERÊNCIAS BIBLIOGRÁFICAS}

ANDRADE, H. S.; OLIVEIRA, V. C.; GONTIJO, T. L.; PESSÔA, M. T. C.; GUIMARÃES, E. A. A. Avaliação do Programa de Controle da Tuberculose: um estudo de caso. Saúde em Debate, Rio de Janeiro, v. 41, n. 01, Mar. de 2017.

ANDRADE, S. L. E. A discursividade de enfermeiros da atenção básica relacionada ao tratamento diretamente observado da tuberculose. 2014. 86 f. Dissertação (Mestrado em Enfermagem) - Universidade Federal da Paraíba, JoãoPessoa, 2014.

BRASIL. Ministério da saúde. Secretaria de vigilância em saúde. Boletim Epidemiológico. V. 50, $n$ 9, Mar. 2019.2 Disponivel em: http://portalarquivos2.saude.gov.br/images/pdf/2019/marco/22/2019-009.pdf. Acesso em: 29 de Dez. de 2019.

BRASIL. Ministério da saúde. Tuberculose ainda é um grave problema de saúdepública. Blog da saúde, 2016. Disponível em: http://www.blog.saude.gov.br/promocao-da-saude/50821tuberculose-ainda-e-um- grave-problema-de-saude-publica.html. Acesso em: 29 de dez. de 2019.

BRUNELLO, M. E. F.; SIMIELE, M. F.; ORFÃO, N. H.; WYSOCK, A. D.;MAGNABOSCO. G. T.; ANDRADE, R. L. P.; MONROE, A. P.; BERALDO, A. A.; VILLA, T. C. S. Atuação da enfermagem na atenção a uma condição crônica(tuberculose): análise de fontes secundárias. Revista Gaúcha de Enfermagem, Porto Alegre, v. 36, n. 01, p. 1-10, Dez, 2015.

CAVALCANTE, E. F.; SILVA, D. M. G. O compromisso do enfermeiro com o cuidado à pessoa com tuberculose. Revista Texto e Contexto Enfermagem, Santa Catarina, v. 25, n. 03, fev. 2016.

COSTA, T. C. Tuberculose na atenção primária à saúde: avaliação dos serviços na perspectiva dos enfermeiros. 2019. 73 f. Dissertação (Mestrado em Enfermagem) Universidade Federal do Amazonas, Manaus, 2019.

FERREIRA, A. F.; QUEIROZ, K. C. S.; TORRES, K. P.; FERREIRA, M. A. F.; ACCIOLY, H.; 
ALVES, M. S. C. F. Os fatores associados à tuberculose pulmonar e a baciloscopia: uma contribuição ao diagnóstico nos serviços de saúde pública. Revista Brasileira de epidemiologia, São Paulo, p. 1-5, jan/jun. 2005.

FIGUEIREDO, J. B. Representações sociais de enfermeiros da estratégia saúde da família sobre o tratamento diretamente observado e de curta duração em tuberculose. 2018. Dissertação (Mestrado em enfermagem) - Universidade Federal deJuiz de Fora, Minas Gerais, 2018.

KOZAVICH. G.; SILVA, R. M.; Tuberculose: Revisão de literatura. Arquivos catarinenses de medicina. Santa Catarina, v. 44, n. 04, dez. 2015.

OLIVEIRA, D. R. C. A prática do enfermeiro da atenção primária à saúde nas consultas ao paciente com tuberculose. 2014. 100f. Dissertação (Mestrado em Enfermagem) - Centro de Ciências da Saúde, Universidade Federal do Rio Grande do Norte, Natal, 2014.

OLIVEIRA, D. R. C. Educação permanente como estratégia para a consulta de enfermagem ao paciente com tuberculose na Atenção Primária à Saúde. 2018. 174f. Tese (Doutorado em Enfermagem na Atenção à Saúde) - Centro de Ciências da Saúde, Universidade Federal do Rio Grande do Norte, Natal, 2018.

OMS pede ação urgente para acabar com a tuberculose. Organização Mundial de Saúde. OPAS Brasil, Brasília, 2018. Disponível em: https://www.paho.org/bra/index.php?option=com_content\&view=article\&id=5760:om s-pedeacao-urgente-para-acabar-com-a-tuberculose\&ltemid=812. Acesso em 29 de Dezembro de 2019.

ORLANDI, G. M. Medidas de apoio ao tratamento da tuberculose: percepção de profissionais de saúde da Atenção Básica no município de São Paulo. 2015. Dissertação (Mestrado em Enfermagem).Universidade de São Paulo, São Paulo, 2017.

PINHEIRO, P. G. O. D. Discursos de profissionais sobre o controle da tuberculose:Pontos de estrangulamento na atenção primária à saúde. 2016.145 f. Dissertação (Mestrado em Enfermagem) - Universidade Federal da Paraíba, JoãoPessoa, 2016.

RABAHI, M. F.; JUNIOR, J. R. L S.; FERREIRA, A. C. G.; SILVA, D. G.; CONDE, M. B. Tratamento da tuberculose. Jornal Brasileiro de Pneumologia, Goiânia, v. 43, n. 05. P. 472486, Maio. 2017.

RÊGO, C. D.; MACÊDO, M. S.; ANDRADE, C. R. B.; MAIA, V. F.; PINTO, J. T. J. M.; PINTO, E. S. G..Processo de trabalho do enfermeiro a pessoa comtuberculose na atenção primária. Revista Baiana de enfermagem, Salvador, v. 29, n. 3, p. 218-228, jul./set. 2015.

RODRIGUES. I. L.; MONTEIRO, L. L.; PACHECO, R. H. B.; SILVA, S. E. D. Abandono do tratamento de tuberculose em co-infectados TB/HIV. Revista da escola de enfermagem da USP, São Paulo, v. 44, n. 02, p. 1-5, jun. 2010.

SILVA, A. E.; SILVA, G. A. O sentido de vivenciar a tuberculose: um estudo sobre representações sociais das pessoas em tratamento. Revista de saúde coletiva, São Paulo, v. 26, n. 04, jun. 2016.

SOUZA, S. S. Apoio às pessoas com tuberculose e redes sociais. 2010. Tese (Doutorado em Enfermagem) - Universidade Federal de Santa Catarina, Florianópolis,2012.

VIEIRA, A. A busca ativa de sintomáticos respiratórios na atenção primária em São Bernardo do Campo. 2016. Dissertação (Mestrado em Enfermagem) - Universidade de São Paulo, São Paulo, 2017. 